\title{
Artificial Soul Optimization - An Invention
}

\author{
${ }^{1}$ Satish Gajawada, ${ }^{2}$ Hassan M.H. Mustafa \\ ${ }^{1}$ Independent Inventor and Scientist \\ Founder and Father of Artificial Human Optimization Field \\ Alumnus, Indian Institute of Technology Roorkee \\ ${ }^{2}$ Faculty of Specified Education, Dept. of Educational Technology, \\ Banha University, Egypt \\ Grand Father of Artificial Human Optimization Field \\ satish.gajawada.iit@gmail.com, prof.dr.hassanmoustafa@gmail.com
}

\begin{abstract}
The Soul is eternal and exists even after death of a person or animal. The main idea that is captured in this work is that soul continues to exist and takes a different body after the death. The primary goal of this work is to invent a new field titled "Artificial Soul Optimization (ASO)". The term "Artificial Soul Optimization" is coined in this paper. All the Optimization algorithms which are proposed based on Artificial Souls will come under "Artificial Soul Optimization" Field (ASO Field). In the Particle Swarm Optimization and Artificial Human Optimization, the basic entities in search space are Artificial Birds and Artificial Humans respectively. Similarly, in Artificial Soul Optimization, the basic entities in search space are Artificial Souls. In this work, the ASO Field concepts are added to Particle Swarm Optimization (PSO) algorithm to create a new hybrid algorithm titled "Soul Particle Swarm Optimization (SoPSO). The proposed SoPSO algorithm is applied on various benchmark functions. Results obtained are compared with PSO algorithm. The World's first Hybrid PSO algorithm based on Artificial Souls is created in this work.

Keywords: Artificial Souls, Artificial Soul Optimization, Artificial Soul Computing, Computational Intelligence, Evolutionary Computing, Particle Swarm Optimization, Genetic Algorithms, Artificial Human Optimization, Bio-Inspired Computing, Nature Inspired Computing, Machine Learning, Artificial Intelligence.
\end{abstract}

\section{Introduction}

The word Soul is present in sacred Hindu religious texts like Srimad Bhagavatham [1] and Bhagavad Gita [2]. "Soul Optimization" is something that deals with Real Souls. It can also be called as "Real Soul Optimization". In this work, the focus is on Artificial Soul Optimization Field (ASO Field) which is defined in the abstract of this paper. Hence it is important to note that "Artificial Soul Optimization" and "Real Soul Optimization" are different.

The corresponding author asked, "Is there something like Soul Computing?" on Researchgate and an expert replied, "Just like I doubt you would find algorithms for 'Unicorn computing', I don't think you will find anything on 'Soul computing'...". Hence there is so much yet to be done in Real Soul Computing and Artificial Soul Computing fields. 
Nikola Tesla said, "The day science begins to study non-physical phenomena, it will make more progress in one decade than in all the previous centuries of its existence". Hence authors would like to suggest scientists to study and do projects related to non-physical phenomena like Real Soul Computing and Artificial Soul Computing. The current work studies Artificial Soul Optimization which comes under Artificial Soul Computing.

New ASO Field algorithms are created in this work by modifying Particle Swarm Optimization (PSO) algorithm with ASO Field concepts. Articles [3-9] give details related to PSO algorithms. Hybrid PSO Algorithms that are created by modifying PSO algorithm were shown in [10-14]. Hybrid PSO algorithms that are created by modifying PSO algorithm with Artificial Human Optimization (AHO) Field concepts and details related to AHO Field are given in articles [15-28]. There are no Artificial Soul Optimization Algorithms (ASO Algorithms) proposed in literature till date. Benchmark Functions used in this paper are taken from [29].

The rest of the article is organized as follows:

Section 2 shows Particle Swarm Optimization algorithm. Section 3 shows "Soul Particle Swarm Optimization (SoPSO)". Results are explained in Section 4. Section 5 gives opportunities that are present in ASO Field. Section 6 gives Conclusions.

\section{Particle Swarm Optimization}

Particle Swarm Optimization (PSO) was proposed by Kennedy and Eberhart in 1995. PSO is based on Artificial Birds. It has been applied to solve complex optimization problems.

In PSO, first we initialize all particles as shown below. Two variables pbest ${ }_{i}$ and gbest are maintained. pbest $_{i}$ is the best fitness value achieved by $i^{\text {th }}$ particle so far and gbest is the best fitness value achieved by all particles so far. Lines 4 to 11 in the below text helps in maintaining particle best and global best. Then the velocity is updated by rule shown in line no. 14 . Line 15 updates position of $\mathrm{i}^{\text {th }}$ particle. Line 19 increments the number of iterations and then the control goes back to line 4 . This process of a particle moving towards its local best and also moving towards global best of particles is continued until termination criteria will be reached.

Procedure: Particle Swarm Optimization (PSO)

1) Initialize all particles

2) iterations $=0$

3) do

4) for each particle i do

5) If $\left(f\left(x_{i}\right)<f\left(\right.\right.$ pbest $\left.\left._{i}\right)\right)$ then

6) $\quad$ pbest $_{i}=x_{i}$

7) end if

8) if $\left(f\left(\right.\right.$ pbest $\left._{i}\right)<f($ gbest $\left.)\right)$ then

9) $\quad$ gbest $=$ pbest $_{i}$

10) end if

11) end for

12) for each particle i do

13) for each dimension d do

14) $\quad v_{i, d}=w^{*} v_{i, d}+$

$\mathrm{C}_{1} *$ Random $(0,1) *\left(\right.$ pbest $\left._{\mathrm{i}, \mathrm{d}}-\mathrm{x}_{\mathrm{i}, \mathrm{d}}\right)$

$+\mathrm{C}_{2}{ }^{*}$ Random $(0,1) *\left(\right.$ gbest $\left._{d}-x_{i, d}\right)$ 
15)

17)

18)

19) iterations $=$ iterations +1

20) while ( termination condition is false)

\section{Soul Particle Swarm Optimization}

The basic entities in Soul Particle Swarm Optimisation (SoPSO) are Artificial Souls. Each Artificial Soul corresponds to a point in search space. For the sake of simplicity, in this work we assume that there are two types of bodies that each Artificial Soul can take. The first body has Body Factor of BF1 and the second body has a Body Factor of BF2. In each generation, Artificial Souls take either first body or second body based on random number generated and BodySelectionProbability. If random number generated is less than BodySelectionProbability then first body is taken else second body is taken. In this study we took BF1 as 0.9 and BF2 as 0.1. Hence if Artificial Soul takes first body then it moves faster in search space because BF1 is 0.9. Whereas if Artificial Soul takes second body it moves slower in the search space because BF2 is 0.1. In each generation, body is dead after velocity and position are updated. Hence Artificial Soul takes new body in next generation. So the Artificial Soul remains eternal in all generations whereas the bodies taken are dead and a new body is taken in every generation by Artificial Souls.

In line number 13, a random number is generated and compared with BodySelectionProbability. If random number is less than BodySelectionProbability then the Soul takes first body else it takes second body. If first body is selected by Soul then lines 14-17 are executed and body factor BF1 is used in the position update equation. If second body is selected then lines 19-22 are executed and body factor BF2 is used in the position update equation. After velocity and position updates, the body taken by Soul is dead. This is the procedure shown for first generation and first Soul. The same procedure is repeated for all the Artificial Souls in first generation. Hence after velocity and position updates, all bodies taken by Souls are dead. Now the second generation is started and Souls take bodies based on random number and BodySelectionProbability as shown in line number 13 . The remaining procedure is same as that of first generation. This process continues until termination criteria will be reached.

Procedure: Soul Particle Swarm Optimization (SoPSO)

1) Initialize all particles

2) iterations $=0$

3) do

4) for each particle i do

5) If $\left(f\left(x_{i}\right)<f\left(\right.\right.$ pbest $\left.\left._{i}\right)\right)$ then

6)

$$
\begin{aligned}
& \text { end if } \\
& \text { if }\left(f\left(\text { pbest }_{i}\right)<f(\text { gbest })\right) \text { then }
\end{aligned}
$$$$
\text { end if }
$$$$
\text { gbest }=\text { pbest }_{i}
$$

end for

$$
\text { for each particle i do }
$$

$$
\text { if ( } \operatorname{rand}(0,1)<\text { BodySelectionProbability) // Soul takes first body }
$$
for each dimension $d$ do

$$
v_{i, d}=w^{*} v_{i, d}+
$$


16)

19)

24)

25)

26) while ( termination condition is false)

$$
\begin{aligned}
& \mathrm{C}_{1} * \text { Random }(0,1) *\left(\text { pbest }_{i, d}-x_{i, d}\right) \\
+ & C_{2} * \operatorname{Random}(0,1) *\left(\text { gbest }_{d}-x_{i, d}\right)
\end{aligned}
$$

end for

else // Soul takes second body

$$
\text { for each dimension d do }
$$

$$
\begin{aligned}
\mathrm{v}_{\mathrm{i}, \mathrm{d}}= & \mathrm{w}^{*} \mathrm{v}_{\mathrm{i}, \mathrm{d}}+ \\
& \mathrm{C}_{1} * \text { Random }(0,1) *\left(\text { pbest }_{\mathrm{i}, \mathrm{d}}-\mathrm{x}_{\mathrm{i}, \mathrm{d}}\right) \\
+ & \mathrm{C}_{2} * \operatorname{Random}(0,1) *\left(\text { gbest }_{\mathrm{d}}-\mathrm{x}_{\mathrm{i}, \mathrm{d}}\right) \\
\mathrm{x}_{\mathrm{i}, \mathrm{d}}= & \mathrm{x}_{\mathrm{i}, \mathrm{d}}+\mathrm{BF2} \mathrm{v}^{*} \mathrm{v}_{\mathrm{i}, \mathrm{d}}
\end{aligned}
$$

end for

$$
\text { end if }
$$

end for

iterations $=$ iterations +1

\section{Results}

The proposed Soul Particle Swarm Optimization (SoPSO) is applied on five benchmark functions. Results obtained are compared with PSO.

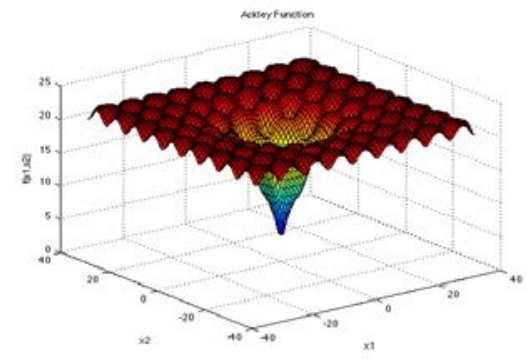

Figure 1. Ackley Function

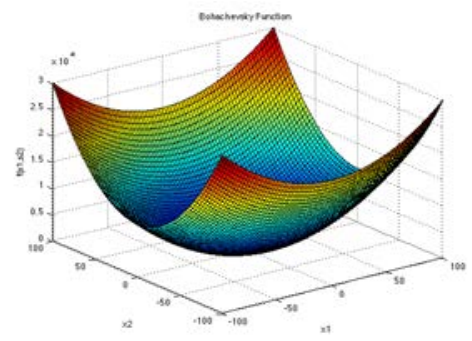

Figure 3. Bohachevsky Function

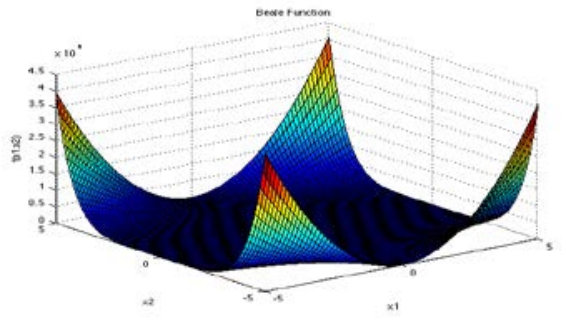

Figure 2. Beale Function

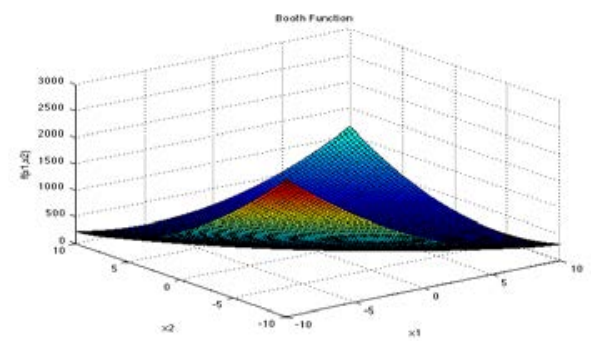

Figure 4. Booth Function

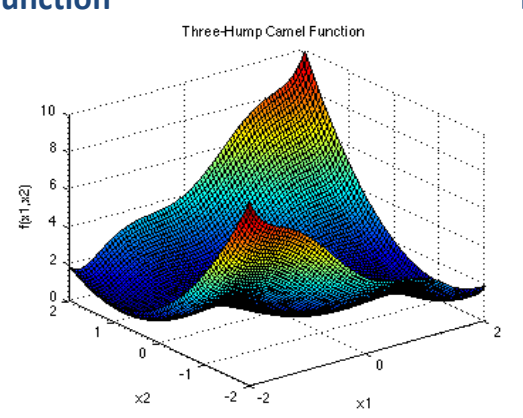

Figure 5. Three-Hump Camel Function 
Table 1. Overall Result

\begin{tabular}{|c|c|c|}
\hline Benchmark Function / Algorithm & SoPSO & PSO \\
\hline Ackley Function & & \\
\hline Beale Function & & \\
\hline Bohachevsky Function & & \\
\hline Booth Function & & \\
\hline Three-Hump Camel Function & & \\
\hline
\end{tabular}

In Table 1 Green represents Performed well. Red represents didn't performed well. Blue represents performed between well and not well. From Table 1 we can see that both SoPSO and PSO performed well on all benchmark functions.

\section{Interesting Opportunities in Artificial Soul Optimization Field}

The following are opportunities for experts in Computational Intelligence Field:

1) International Institute of Artificial Soul Optimization, Hyderabad, INDIA

2) Indian Institute of Technology Roorkee Artificial Soul Optimization Labs, IIT Roorkee

3) Foundation of Artificial Soul Optimization, New York, USA.

4) IEEE Artificial Soul Optimization Society

5) ELSEVIER journals in Artificial Soul Optimization

6) Applied Artificial Soul Optimization - A New Subject

7) Advanced Artificial Soul Optimization - A New Course

8) Invited Speech on "Artificial Soul Optimization" in world class Artificial Intelligence Conferences

9) A Special issue on "Artificial Soul Optimization" in a Springer published Journal

10) A Seminar on "Recent Advances in Artificial Soul Optimization" at Technical Festivals in colleges

11) International Association of Artificial Soul Optimization (IAASO)

12) Transactions on Artificial Soul Optimization (TASO)

13) International Journal of Artificial Soul Optimization (IJASO)

14) International Conference on Artificial Soul Optimization (ICASO)

15) www.ArtificialSoulOptimization.com

16) B.Tech in Artificial Soul Optimization

17) M.Tech in Artificial Soul Optimization

18) $\mathrm{PhD}$ in Artificial Soul Optimization

19) PostDoc in Artificial Soul Optimization

20) Artificial Soul Optimization Labs

21) To become "Father of Artificial Soul Optimization" field 


\section{Conclusions}

A new field titled "Artificial Soul Optimization (ASO)" is invented in this work. A new algorithm titled Soul Particle Swarm Optimization (SoPSO) is designed and results show that proposed SoPSO performed well on all benchmark functions like PSO. In this work, a list of opportunities in ASO Field is shown for Computational Intelligence Field Experts. Not much work was done in Soul Computing and Artificial Soul Computing Fields till date. The new ASO field invented in this work comes under Artificial Soul Computing Field. There is scope for other innovative algorithms like Soul Ant Colony Optimization (SoACO) similar to SoPSO.

\section{ACKNOWLEDGEMENTS}

The authors of this work would like to thank the following Editorial Members of "Transactions on Machine Learning and Artificial Intelligence (TMLAI)" for accepting our innovative invention titled Artificial Soul Optimization Field (ASO Field):

Professor Er Meng Joo, School of Electrical and Electronic Engineering, Nanyang Technological University, Singapore

Professor Djamel Bouchaffra, Department of Mathematics and Computer Science, Grambling State University, Louisiana., United States

Prof Bhavani Thuraisingham, Department of Computer Science, Erik Jonsson School of Engineering and Computer Science, The University of Texas at Dallas, United States

Professor Dong-Hee Shin, Department of Interaction Science, Social Computing Institute, Sungkyunkwan University, Seoul, Korea, Republic of

Professor Filippo Neri, Professor of Computer Information Systems, Faculty of Information \& Communication Technology, Dar Guzeppi Zahra, University of Malta, Malta

Prof Mohamed A Zohdy, Department of Electrical and Computer Engineering, Oakland University, United States

Professor Wee SER, Associate Professor Director, VALENS Research Centre, School of Electrical and Electronic Engineering; Program Director (MISA), Nanyang Institute of Technology in Medicine and Health Nanyang Technological University, Singapore

Professor Liang Yanchun, Deputy Dean of College of Computer Science, Jilin University, China

Professor Julia Johnson, Dept. of Mathematics \& Computer Science Laurentian University, Sudbury, Ontario, Canada

Professor Farouk Yalaoui, Institut Charles Dalaunay, University of Technology of Troyes, France

Professor Jai N Singh, Department of Mathematics and Computer Science, Barry University, Miami Shores, Florida, United States

Dr Kyriakos G Vamvoudakis, Dept of Electrical and Computer Engineering, University of California Santa Barbara, United States 
Satish Gajawada, Hassan M.H. Mustafa; Artificial Soul Optimization - An Invention. Transactions on Machine Learning and Artificial Intelligence, Volume 7 No 5 October (2019); pp: 36-44

Dr Zezhi Chen, School of Computing and Information Systems; Faculty of Science, Engineering and Computing; Kingston University London, United Kingdom

Dr Luis Rodolfo Garcia, College of Science and Engineering, Texas A\&M University, Corpus Christi, United States

Dr Hafiz M. R. Khan, Associate Professor \& Graduate Program Director, Department of Biostatistics, Florida International University, United States

Dr Xiaocong Fan, School of Engineering, Behrend College, The Pennsylvania State University, United States

Dr Chen Yanover, Machine Learning for Healthcare and Life Sciences IBM Haifa Research Lab, United States

Dr Vandana Janeja, Information Systems Department, University of Maryland, Baltimore, United States

Dr Nikolaos Georgantas, Senior Research Scientist at INRIA, Paris-Rocquencourt, France

Dr Zeyad Al-Zhour, College of Engineering, The University of Dammam, Saudi Arabia

Dr Zdenek Zdrahal, Senior Research Fellow in Artificial Intelligence, Knowledge Media Institute, The Open University, Milton Keynes MK7 6AA, United Kingdom

Dr Steve S. H. Ling, University of Technology, Sydney, Australia

Dr Marianne Hoogeveen, Arena Analytics, United States

Dr Hooshang Hemami, Dept. of Electrical and Computer Engineering The Ohio State University Columbus, Ohio 43210, United States

Dr Hiroyuki Utsunomiya, University of Tokyo, Japan

\section{REFERENCES}

[1] Veda Vyasa, Srimad Bhagavatha Purana. Ancient Hindu Religious Text.

[2] Veda Vyasa, Bhagavad Gita. Ancient Hindu Religiuos Text.

[3] Saptarshi Sengupta, Sanchita Basak, Richard Alan Peters II. Particle Swarm Optimization: A survey of historical and recent developments with hybridization perspectives. https://arxiv.org/abs/1804.05319, 2018.

[4] Yudong Zhang, Shuihua Wang, and Genlin Ji, "A Comprehensive Survey on Particle Swarm Optimization Algorithm and Its Applications," Mathematical Problems in Engineering, vol. 2015, Article ID 931256, 38 pages, 2015. https://doi.org/10.1155/2015/931256.

[5] M. R. AlRashidi, M. E. El-Hawary. A Survey of Particle Swarm Optimization Applications in Electric Power Systems. IEEE Transactions on Evolutionary Computation. Volume 13, Issue 4, August 2009.

[6] Sharandeep Singh. A Review on Particle Swarm Optimization Algorithm. International Journal of Scientific \& Engineering Research, Volume 5, Issue 4, April-2014.

[7] T. Saravanan and V. Srinivasan. Overview of Particle Swarm Optimization. Indian Journal of Science and Technology, Vol 8(32), November 2015.

[8] Muhammad Imran, Rathiah Hashim, Noor Elaiza Abd Khalid.An Overview of Particle Swarm Optimization Variants. Procedia Engineering. Elsevier.Volume 53, Pages 491-496, 2013. 
[9] Riccardo Poli, James Kennedy, Tim Blackwell. Particle swarm optimization - An overview. Swarm Intelligence. Volume 1, Issue 1, pp 33-57, Springer, 2007.

[10] Liu H, Xu G, Ding GY, Sun YB, "Human behavior-based particle swarm optimization”, The Scientific World Journal, 2014.

[11] Ruo-Li Tang, Yan-Jun Fang, "Modification of particle swarm optimization with human simulated property", Neurocomputing, Volume 153, Pages 319-331, 2015.

[12] Muhammad Rizwan Tanweer, Suresh Sundaram, "Human cognition inspired particle swarm optimization algorithm", 2014 IEEE Ninth International Conference on Intelligent Sensors, Sensor Networks and Information Processing (ISSNIP), 2014.

[13] M.R. Tanweer, S. Suresh, N. Sundararajan, "Self regulating particle swarm optimization algorithm", Information Sciences: an International Journal, Volume 294, Issue C, Pages 182-202, 2015.

[14] M. R. Tanweer, S. Suresh, N. Sundararajan, "Improved SRPSO algorithm for solving CEC 2015 computationally expensive numerical optimization problems", 2015 IEEE Congress on Evolutionary Computation (CEC), pp. 19431949, 2015.

[15] Satish Gajawada. "POSTDOC : The Human Optimization", Computer Science \& Information Technology (CS \& IT), CSCP, pp. 183-187, 2013.

[16] Satish Gajawada. "CEO: Different Reviews on PhD in Artificial Intelligence", Global Journal of Advanced Research, vol. 1, no.2, pp. 155-158, 2014.

[17] Satish Gajawada. "Entrepreneur: Artificial Human Optimization". Transactions on Machine Learning and Artificial Intelligence, Volume 4 No 6 December (2016); pp: 64-70

[18] Satish Gajawada. "Artificial Human Optimization - An Introduction", Transactions on Machine Learning and Artificial Intelligence, Volume 6, No 2, pp: 1-9, April 2018

[19] Satish Gajawada. "An Ocean of Opportunities in Artificial Human Optimization Field", Transactions on Machine Learning and Artificial Intelligence, Volume 6, No 3, June 2018

[20] Satish Gajawada. "25 Reviews on Artificial Human Optimization Field for the First Time in Research Industry", International Journal of Research Publications, Vol. 5, no. 2, United Kingdom.

[21] Satish Gajawada and Hassan M. H. Mustafa, "Collection of Abstracts in Artificial Human Optimization Field", International Journal of Research Publications, Volume 7, No 1, United Kingdom, 2018.

[22] Satish Gajawada, Hassan M. H. Mustafa, HIDE : Human Inspired Differential Evolution - An Algorithm under Artificial Human Optimization Field, International Journal of Research Publications (Volume: 7, Issue: 1), http://ijrp.org/paper-detail/264

[23] Satish Gajawada, Hassan M. H. Mustafa , Artificial Human Optimization - An Overview. Transactions on Machine Learning and Artificial Intelligence, Volume 6, No 4, August 2018.

[24] Satish Gajawada, Hassan M. H. Mustafa, Testing Multiple Strategy Human Optimization based Artificial Human Optimization Algorithms, Computer Reviews Journal, vol. 1, no.2, 2018.

[25] Satish Gajawada, Hassan M. H. Mustafa. Hybridization Concepts of Artificial Human Optimization Field Algorithms Incorporated into Particle Swarm Optimization. International Journal of Computer Applications 181(19):10-14, September 2018.

[26] Satish Gajawada, Hassan M. H. Mustafa (2018). An Artificial Human Optimization Algorithm Titled Human Thinking Particle Swarm Optimization. International Journal of Mathematical Research, 7(1): 18-25. DOI: 10.18488/journal.24.2018.71.18.25 
Satish Gajawada, Hassan M.H. Mustafa; Artificial Soul Optimization - An Invention. Transactions on Machine Learning and Artificial I ntelligence, Volume 7 No 5 October (2019); pp: 36-44

[27] Satish Gajawada, Hassan Mustafa: Novel Artificial Human Optimization Field Algorithms - The Beginning. CoRR abs/1903.12011(2019)

[28] ] Satish Gajawada, Hassan M. H. Mustafa: Ten Artificial Human Optimization Algorithms. Transactions on Machine Learning and Artificial Intelligence, Volume 7, No 3, June 2019.

[29] https://www.sfu.ca/ ssurjano/optimization.html 